\section{Traumatische Erlebnisse erhöhen die Gewaltbereitschaft}

_ Nach einem traumatischen Ereignis, einer Selbstverletzung oder einer Substanzintoxikation ist die Gewaltbereitschaft eines Menschen vorübergehend erhöht. Dass dies nicht nur für psychisch Kranke gilt, wurde in einer schwedischen Kohorte deutlich.

Forscher der Universität Oxford entnahmen dem nationalen schwedischen Patientenregister Daten von 34.903 Personen mit Schizophrenie-SpektrumStörungen, 29.692 Patienten mit bipolaren Störungen und 2.763.012 psychisch gesunden Kontrollpersonen. In jeder Gruppe identifizierten sie die Personen, die einem der folgenden Trigger ausge- setzt gewesen waren: Opfer einer Gewalthandlung, Verlust der Eltern, Selbstverletzung, Hirntrauma, unbeabsichtigte Verletzungen, Drogen- bzw. Alkoholintoxikation. Danach verglichen sie mittels logistischer Regression das postexpositionelle Risiko, gewalttätig zu werden, mit der Gewaltbereitschaft in einem entsprechenden triggerfreien früheren Zeitraum (Kontrollperiode).

In allen Gruppen zeichnete sich in der Woche nach einem Triggerereignis erhöhte Gewaltbereitschaft ab. Die meisten Taten wurden verübt, wenn der Täter in der Woche zuvor selbst ein Gewalterlebnis hatte.

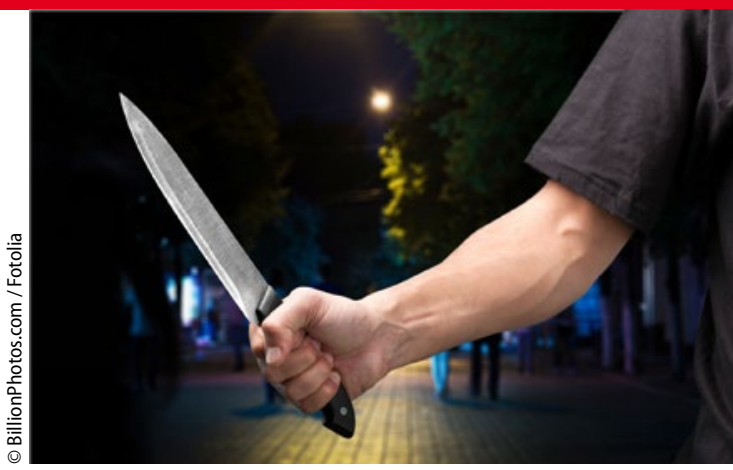

Dabei ergaben sich keine nennenswerten Unterschiede zwischen psychisch kranken Patienten und Personen der Kontrollgruppe. Nur der Verlust der Eltern hatte bei Schizophrenie-Patienten deutlich stärkere Auswirkungen als bei psychisch Gesunden.

Die Untersuchung zeige, so die Autoren, dass Interventionen zur Gewaltreduktion nach einem Unfall oder Drogenrausch nicht nur für psychiatrische Patienten, sondern für jeden sinnvoll seien. $\quad$ - st

- Sariaslan A et al. JAMA Psychiatry. Published online July 13, 2016. doi:10.1001/jamapsychiatry.2016.1349.

\section{Früh zu Bett mit 5 - schlank mit 15}

Wer im Kindergartenalter nach 20 Uhr noch wach ist, hat später ein erhöhtes Adipositasrisiko.

Forscher der Ohio State University analysierten Daten von 977 Teilnehmern der Study of Early Child Care and Youth Development. Die Schlafenszeit der Kinder im Vorschulalter wurde zehn Jahre später mit ihrem BMI in Beziehung gesetzt.

$25 \%$ der Kinder waren bis spätestens $20 \mathrm{Uhr}$ im Bett, $50 \%$ zwischen 20 und 21 Uhr und $25 \%$ gingen nach 21 Uhr schlafen. Als Jugendliche waren $16 \%$ der Probanden adipös. Dabei ergab sich ein klarer Zusammenhang zu den Schlafenszeiten im Kindergartenalter. Nur jeder zehnte Jugendliche, der als Kind bis spätestens 20 Uhr im Bett war, war mit 15 Jahren adipös. Von denen, die zwischen 20 und $21 \mathrm{Uhr}$ schlafen gegangen waren, legten $16 \%$ zu viel Gewicht zu, und wer schon in jüngsten Jahren Open-end-Bettgehzeiten hatte, dessen Adipositasrisiko war mit $23 \%$ am höchsten. Für Frühschläfer gegenüber Spätschläfern errechnete sich ein relativer Vorteil von $52 \%$.

wt 